\title{
Embedded Polar Quantization
}

\author{
Emmanuel Ravelli, Student Member, IEEE, and Laurent Daudet, Member, IEEE
}

\begin{abstract}
Embedded polar quantization can be useful for progressive transmission of circularly symmetric data, e.g., for fine-grain scalable coding of parametric audio. Sets of constrained-resolution embedded quantizers are built recursively by successive refinement processes, that are detailed for strict polar quantization and unrestricted polar quantization. The quadratic error minimization problem is solved using equations similar to those of Max, and the refinement algorithm can, in the unrestricted case, be simplified using a high-rate approximation. For Gaussian data, comparisons with reference non-embedded quantizers show that the embedding property comes at an often negligible cost in terms of rate-distortion performance.
\end{abstract}

Index Terms-Embedded quantization, quantizer design, scalable audio coding.

\section{INTRODUCTION}

$\mathbf{C}$ YONSTRAINED-RESOLUTION embedded quantization as stated in this letter can be defined as follows. Consider K quantizers $\mathcal{Q}^{(k)}, k=1, \ldots, K$

$$
\mathcal{Q}^{(k)}: \mathbf{R}^{L} \rightarrow\left\{0, \ldots, 2^{k}-1\right\}
$$

with $L$ the dimension of the sample space and $N^{(k)}=2^{k}$, the number of cells for quantizer $\mathcal{Q}^{(k)}$. The output of the quantizer $\mathcal{Q}^{(k)}$ is a fixed-length $k$-bits binary code

$$
c_{1}^{(k)} c_{2}^{(k)} c_{3}^{(k)} \ldots c_{k}^{(k)} \text {, with } c_{1}^{(k)}, \ldots, c_{k}^{(k)} \in\{0,1\} .
$$

The quantizers are said to be embedded if there exists fixedlength binary codes such that

$$
c_{l}^{(k)}=c_{l}^{(k+1)}, \text { for } 1 \leq k<K, \text { and } 1 \leq l \leq k .
$$

In other words, the quantizers are embedded if they produce a sequence of embedded binary codes: higher rate codes contain lower rate codes plus bits of refinement.

Embedded quantizers find useful applications in progressive transmission of information. A well-known example is scalable image coding (e.g., EZW [1], SPIHT [2]), where wavelet coefficients are quantized using scalar $(L=1)$ embedded quantizers. As the number of received bits increases, the images are progressively displayed with more and more detail; this property is commonly used in web browsing. Although not as widespread, a similar application can be found for scalable audio coding. One can imagine streaming the same audio content to several users with different and/or varying bandwidth possibilities. With standard audio coding, the server has to store a number of bitstreams corresponding to different compression ratio, and for a given

Manuscript received September 14, 2006; revised February 17, 2007. The associate editor coordinating the review of this manuscript and approving it for publication was Dr. Stefano Buzzi.

The authors are with the Université Pierre et Marie Curie-Paris 6, Institut Jean Le Rond d'Alembert-LAM, 75015 Paris, France (e-mail: ravelli@lam. jussieu.fr; daudet@lam.jussieu.fr).

Digital Object Identifier 10.1109/LSP.2007.896379 user, it must select the one corresponding to its bandwidth using a conservative approach. In the case of embedded coding, a bitstream at maximum resolution is stored; users with less bandwidth just need to stop the reception at any time, yet they can still receive the file with the best quality given their bandwidth.

Recent work [3] focused on applying the SPIHT algorithm to MDCT-based audio coding. Transform coding gives excellent results at high rates, but at low rates, the quality decreases significantly, and thus, parametric coding performs better. While MDCT-based coding uses real coefficients and thus scalar quantization, some parametric representations (e.g., [4]) produce complex coefficients. Each complex coefficient is efficiently represented in polar form: its norm and angle are the amplitude and the phase. Another application is image coding using complex wavelet transforms [5]. These kinds of representations imply the need for an efficient polar quantizer that has also the embedding property. This letter addresses this problem.

Embedded quantization was first introduced for scalar quantization in [6]. An optimal quantizer is chosen first as a reference; it is designed using the standard numerical optimization methods of Max [7]. The quantizers of lower and higher rates are designed by iteratively aligning the quantization thresholds and optimizing the thresholds and reconstruction points using methods similar to [7]. Such quantizers can be easily represented using binary trees. Each stage of the tree corresponds to a particular rate, the nodes correspond to the boundary points, and the branches correspond to the output levels. Practical embedded scalar quantizers have also been proposed in [8]-[10]. Finally, embedded quantization has been generalized to vector quantization. An example of such vector quantizers are the treestructured vector quantizers (TSVQ) [11]. The 2-ary TSVQ is designed using a binary tree and numerical solving mehods similar to [7]; the embedded quantizers are then the pruned tree corresponding to the first $k$ stages of the TSVQ.

This letter considers a particular case of two-dimensional $(L=2)$ quantizers that enable embedded polar quantization. Polar quantizers are natural quantizers for two-dimensional data with circularly symmetric densities. Amplitude and phase can be quantized separately, in this case is called strict polar quantization (SPQ [12]), or they can be quantized jointly, called unrestricted polar quantization (UPQ [13]). In this letter, a circularly symmetric complex variable $x$ is used, represented by its polar coordinates $(r, \theta)$. Since amplitude and phase variables are independent, the variable joint density function is then

$$
f_{X}(r, \theta)=\frac{1}{2 \pi} f_{R}(r)
$$

where $f_{R}(r)$ is the marginal density function of the amplitude variable. A polar quantizer $\mathcal{Q}^{(k)}$ with $N^{(k)}$ cells is defined as follows. The amplitude range is partitioned into $M^{(k)}$ levels and, within each amplitude level, indexed by $m=1,2, \ldots, M^{(k)} ;$ there are $P_{m}^{(k)}$ equal-sized phase cells, such that $\sum_{m=1}^{M^{(k)}} P_{m}^{(k)}=N^{(k)}$. Note that for SPQ, one has 
$P_{m}^{(k)}=P^{(k)}$ for all $m$. The boundaries of the amplitude levels are

$$
R_{0}^{(k)}=0<R_{1}^{(k)}<R_{2}^{(k)}<\cdots<R_{M}^{(k)}=\infty
$$

and the boundaries of the phase regions for the amplitude level $m$ are

$$
0<\frac{2 \pi}{P_{m}^{(k)}}<2 \frac{2 \pi}{P_{m}^{(k)}}<\cdots<\left(P_{m}^{(k)}-1\right) \frac{2 \pi}{P_{m}^{(k)}}<2 \pi .
$$

The output point for the cell $\mathcal{R}_{m, p}^{(k)}$ defined by the amplitude level $m$ and the phase region $p$ is $\left(\alpha_{m}^{(k)}, \beta_{m, p}^{(k)}\right)$. The mean-square error is then expressed by

$$
D^{(k)}=\sum_{m=1}^{M^{(k)}} \sum_{p=1}^{P_{m}^{(k)}} \iint_{\mathcal{R}_{m, p}^{(k)}}\left|r e^{j \theta}-\alpha_{m}^{(k)} e^{j \beta_{m, p}^{(k)}}\right|^{2} f_{X}(r, \theta) d r d \theta .
$$

The purpose of this letter is to detail simple construction rules for embedded quantizers, in the cases of SPQ (see Section II) and UPQ (see Section III), and to compare the performance of the embedded quantizers to the performance of the non-embedded quantizers for Gaussian data.

\section{EMbedded Strict Polar QuANTIZATION}

\section{A. Quantizers Design}

SPQ is first considered. As amplitude and phase are quantized separately, scalar embedded quantization techniques can easily be used to design the embedded SPQ. An algorithm similar to [6] is used, where an optimal reference quantizer is first selected. Then, successive lower- and higher-rate quantizers are built in a recursive manner. The design algorithm consists of the following three steps:

1) Reference quantizer: An optimal SPQ $\mathcal{Q}^{\left(k_{r e f}\right)}$ is designed using the algorithm of [12]. $\mathcal{Q}^{\left(k_{r e f}\right)}$ has a number of amplitude levels $M^{\left(k_{r e f}\right)}$ and a number of phase levels $P^{\left(k_{r e f}\right)}$.

2) Lower-rate quantizers: Two lower-rate quantizers $\mathcal{Q}^{(k)}$ are designed from $\mathcal{Q}^{(k+1)}$. The first one is designed from $\mathcal{Q}^{(k+1)}$ by selecting only every second amplitude boundary and keeping the same number of phase levels. The other one is designed from $\mathcal{Q}^{(k+1)}$ by selecting only every second phase boundary and keeping the same number of amplitude levels. The output points for the two quantizers are found using

$$
\begin{aligned}
\alpha_{m}^{(k)} & =S\left(P^{(k)}\right) \frac{\int_{R_{m-1}^{(k)}}^{R_{m}^{(k)}} r f_{R}(r) d r}{\int_{R_{m-1}^{(k)}}^{R_{(k)}^{(k)}} f_{R}(r) d r} \\
\beta_{m, p}^{(k)} & =\left(p-\frac{1}{2}\right) \frac{2 \pi}{P^{(k)}}
\end{aligned}
$$

with $S\left(P^{(k)}\right)=\operatorname{sinc}\left(\pi / P^{(k)}\right)$. Then, the distortion is computed for each quantizer using (7), and the one with the minimum distortion is selected.

3) Higher-rate quantizers: Similarly, two higher-rate quantizers $\mathcal{Q}^{(k)}$ are designed from $\mathcal{Q}^{(k-1)}$. The first one is designed from $\mathcal{Q}^{(k-1)}$ by refining the amplitude quantizer by two and keeping the same number of phase levels. The new amplitude
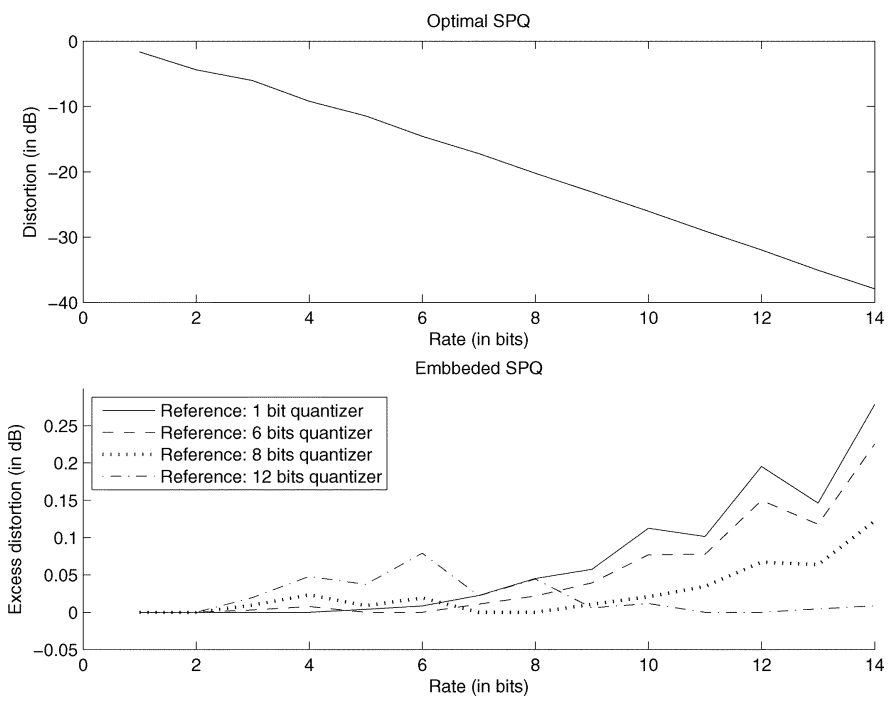

Fig. 1. (Top) Rate-distortion curve for the optimal SPQ of [12]. (Bottom) Excess distortion over the optimal SPQ for several embedded SPQs using different values for $k_{r e f}$.

boundaries are initialized at the middle of the previous amplitude boundaries. Boundaries and output points are then computed iteratively by first updating the output points using (8) and (9) and next by updating the new amplitude boundaries using

$$
R_{m}^{(k)}=\frac{1}{S\left(P^{(k)}\right)} \frac{\alpha_{m}^{(k)}+\alpha_{m+1}^{(k)}}{2}
$$

Another higher-rate quantizer can be designed from $\mathcal{Q}^{(k-1)}$ by refining the phase quantizer by two and keeping the same number of amplitude levels. The new phase boundaries are at the middle of the previous boundaries, and the new output points are calculated using (8) and (9). Again, the distortion is computed for each quantizer using (7), and the one with the minimum distortion is selected.

\section{B. Application: Gaussian Data}

The performance of our quantizers is evaluated using an independent bivariate Gaussian source. Such a source gives a good base of comparison as the results for existing quantizers are well known [12], [13]. Moreover, the source produced by the DFT of a stationary signal of length $\mathrm{N}$ tends to a bivariate Gaussian source as $\mathrm{N}$ becomes large. A bivariate Gaussian source is equivalent to an independent circularly symmetric complex variable whose amplitude has a Rayleigh distribution. The joint density function is then $f_{X}(r, \theta)=(1 / 2 \pi) f_{R}(r)$ with $f_{R}(r)=\left(r / \sigma^{2}\right) \exp \left(-r^{2} / 2 \sigma^{2}\right)$. Several embedded SPQs are compared using different values for $k_{r e f}$ and these embedded SPQs are also compared with the (non-embedded) optimal SPQ (from [12]).

Fig. 1 shows the results obtained for $k_{\text {ref }}=1,6,8,12$ bits. When the actual number of bits is not far from $k_{r e f}$, the excess distortion of the embedded quantizers is low, typically less than $0.2 \mathrm{~dB}$. The choice of $k_{r e f}$ will depend on the application: a low $k_{r e f}$ is selected if the probability of having low rates is high; and a high $k_{r e f}$ is selected if the probability of having low rates is low. Note that in cases where little is known a priori about such probability, a conservative approach should favor a large $k_{r e f}$. 


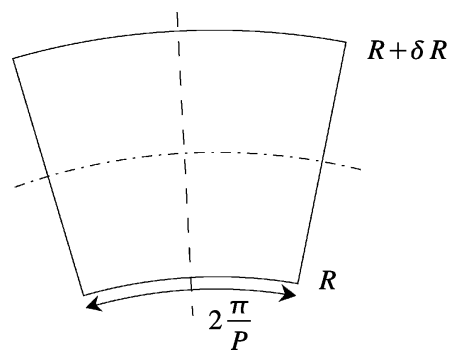

Fig. 2. Cell delimited by the amplitude boundaries $R$ and $R+\delta R$ and the phase boundaries difference $2 \pi / P$. The dashed line represents the new cells boundary for phase refinement, and the dash-dotted line represents the new cells boundary for amplitude refinement.
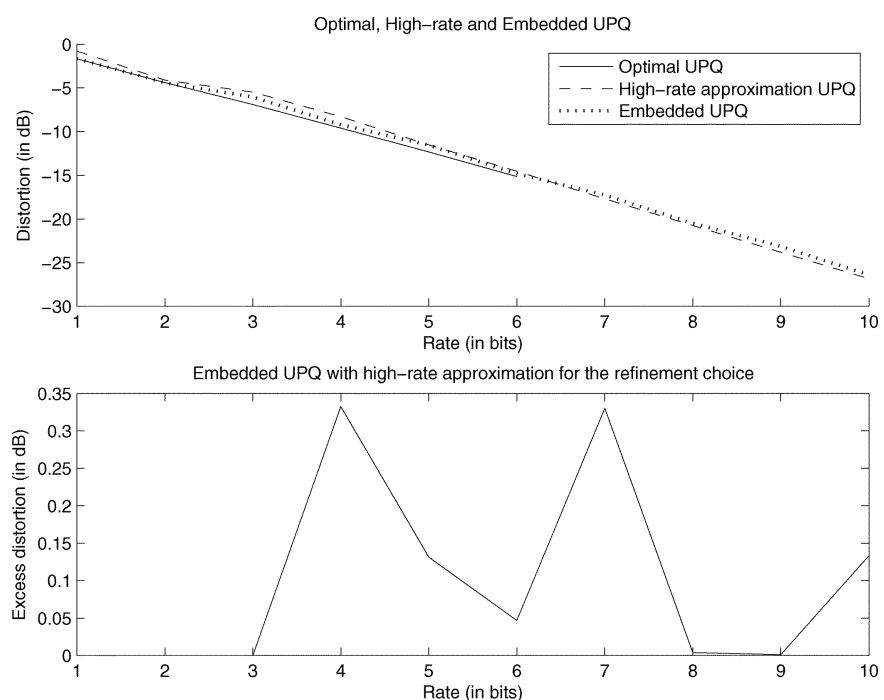

Fig. 3. (Top) Rate-distortion curves for the optimal UPQ of [13], the optimal high-rate UPQ of [14], and our embedded UPQ (without the high-rate approximation for the refinement choice). (Bottom) Excess distortion over the embedded UPQ for the embedded UPQ with the high-rate approximation for the refinement choice.

\section{EMBEDDED UnRestricted POLAR QUANTIZATION}

\section{A. Quantizers Design}

Consider the more general case of unrestricted polar quantization (UPQ). Here, lower-rate embedded quantizers cannot necessarily be found using an optimal reference quantizer, and consequently, the same design method as in SPQ cannot be used. Indeed, an optimal UPQ as designed in [13] does not necessarily have a power-of-two number of phase regions at a given amplitude level. Instead, an algorithm similar to 2-ary TSVQ is used. The design algorithm consists of the following two steps:

1) Reference quantizer: The first quantizer $\mathcal{Q}^{(1)}$ is the optimal 2-cell quantizer (top-left of Fig. 4).

2) Higher-rate quantizers: For a higher-rate quantizer $\mathcal{Q}^{(k)}$ with $k=2, \ldots, K$, each region of $\mathcal{Q}^{(k-1)}$ is refined separately in two different ways, by refining either the amplitude or the phase. This leads to $2^{2^{k-1}}$ possibilities for $\mathcal{Q}^{(k)}$. However, as the source is supposed to be circularly symmetric, cells at the same amplitude level perform in the same way. Consequently, there are only $2^{M^{(k-1)}}$ possibilities for $\mathcal{Q}^{(k)}$. For a cell at a given amplitude level, one has to decide whether an amplitude
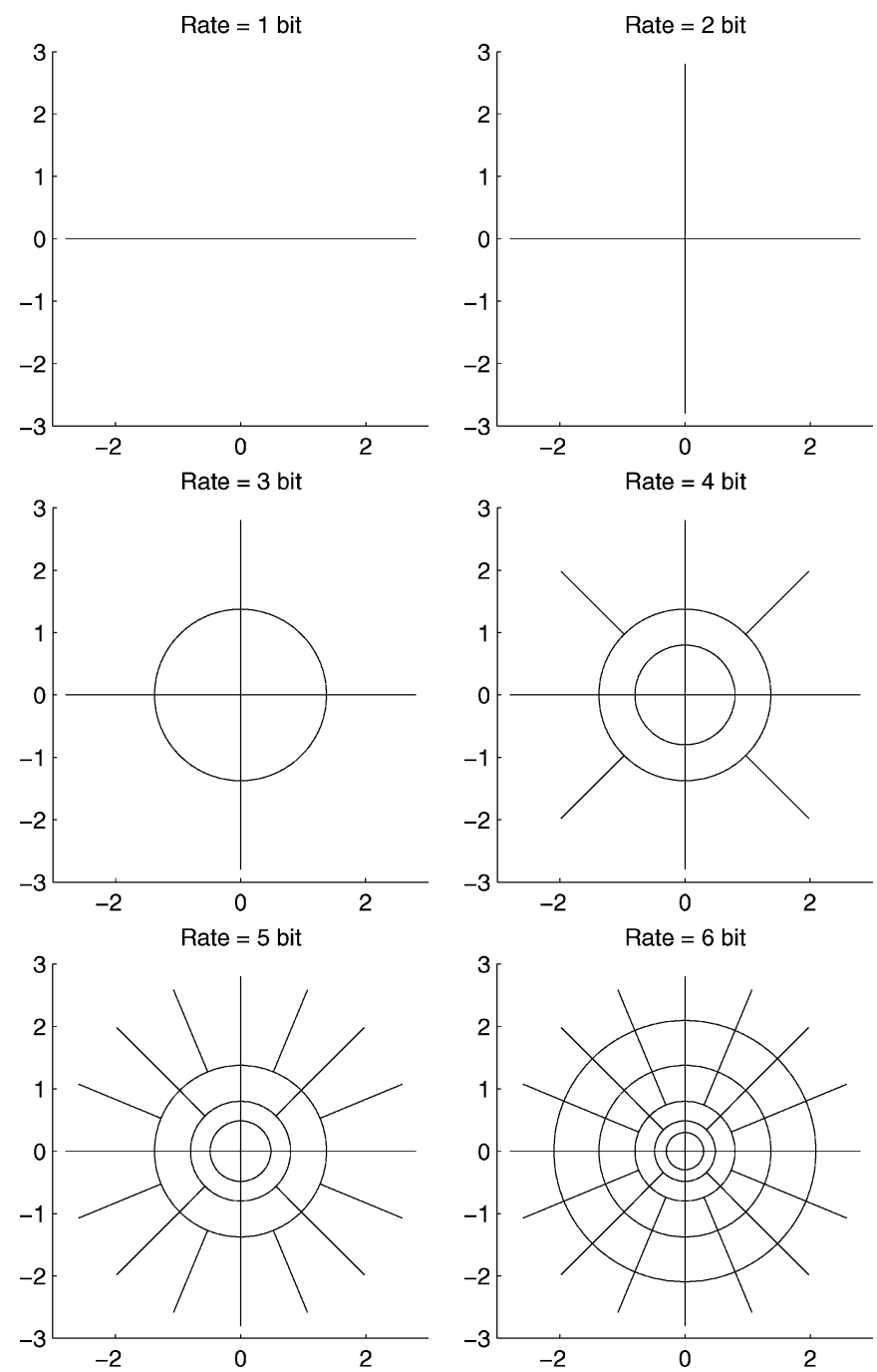

Fig. 4. First six embedded UPQs for Gaussian data.

refinement or a phase refinement gives the smallest distortion; this choice is discussed in the next subsection. Then, the new boundaries and output points are computed using equations similar to those of Max [7]. The same process is repeated for each amplitude level.

\section{B. Choice Between Amplitude and Phase Refinement}

Consider a cell delimited by the amplitude boundaries $R$ and $R+\delta R$ and the phase boundaries difference $2 \pi / P$ (see Fig. 2). Depending on these three parameters, it may be better to choose either an amplitude refinement (the new devided cell is noted (a)) or a phase refinement (the new divided cell is noted (b)). For amplitude refinement, the amplitude boundary $R+\gamma$ and the two new output points $\alpha_{1}$ and $\alpha_{2}$ are calculated iteratively using the following equations:

$$
\begin{aligned}
R+\gamma & =\frac{1}{S(P)} \frac{\alpha_{1}+\alpha_{2}}{2} \\
\alpha_{1} & =S(P) \frac{\int_{R}^{R+\gamma} r f_{R}(r) d r}{\int_{R}^{R+\gamma} f_{R}(r) d r} \\
\alpha_{2} & =S(P) \frac{\int_{R+\gamma}^{R+\delta R} r f_{R}(r) d r}{\int_{R+\gamma}^{R+\delta R} f_{R}(r) d r} .
\end{aligned}
$$


For the phase refinement, the phase boundary is at the middle of the cell, and the new output point $\alpha$ is calculated using

$$
\alpha=S(2 P) \frac{\int_{R}^{R+\delta R} r f_{R}(r) d r}{\int_{R}^{R+\delta R} f_{R}(r) d r} .
$$

The distortions $D_{a}$ and $D_{b}$ are computed using (7), and the one that gives the smallest distortion is selected.

An analytical solution for the refinement choice can be derived using high-rate approximation. The joint probability density function is supposed to be constant in the cell. Moreover, $P$ is supposed to be large, and $S(P)$ is approximated using its second-order Taylor expansion. The total distortion $D_{a}$ for the cell (a) is

$$
\begin{aligned}
D_{a} \cdot \frac{P}{f_{R}(R)}= & \frac{\left(R+\delta R-\alpha_{2}\right)^{3}-\left(R+\frac{\delta R}{2}-\alpha_{2}\right)^{3}}{3} \\
& +\frac{\pi^{2}}{3 P^{2}}\left((R+\delta R)^{2}-\left(R+\frac{\delta R}{2}\right)^{2}\right) \frac{\alpha_{2}}{2} \\
& +\frac{\left(R+\frac{\delta R}{2}-\alpha_{1}\right)^{3}-\left(R-\alpha_{1}\right)^{3}}{3} \\
& +\frac{\pi^{2}}{3 P^{2}}\left(\left(R+\frac{\delta R}{2}\right)^{2}-R^{2}\right) \frac{\alpha_{1}}{2}
\end{aligned}
$$

with

$$
\begin{aligned}
& \alpha_{1}=\left(1-\frac{\pi^{2}}{6 P^{2}}\right)\left(R+\frac{\delta R}{4}\right) \\
& \alpha_{2}=\left(1-\frac{\pi^{2}}{6 P^{2}}\right)\left(R+3 \frac{\delta R}{4}\right) .
\end{aligned}
$$

The total distortion $D_{b}$ for the cell (b) is

$$
\begin{aligned}
D_{b} \cdot \frac{P}{f_{R}(R)}=\frac{(R+\delta R-\alpha)^{3}}{3} & -(R-\alpha)^{3} \\
& +\frac{\pi^{2}}{3(2 P)^{2}}\left((R+\delta R)^{2}-R^{2}\right) \frac{\alpha}{2}
\end{aligned}
$$

with

$$
\alpha=\left(1-\frac{\pi^{2}}{6(2 P)^{2}}\right)\left(R+\frac{\delta R}{2}\right) .
$$

To find the boundaries, $D_{a} \leq D_{b}$ is solved. Using a firstorder approximation of the former expressions, this inequality is equivalent to

$$
\frac{P}{2 \pi} \geq\left(\frac{R}{\delta R}+\frac{1}{2}\right) .
$$

Equation (16) has a simple interpretation: if the length $\delta R$ of the radial boundary of the cell is bigger than $(R+\delta R / 2)(2 \pi / P)$, which is the length of the average circle arc, then amplitude refinement is preferred, and vice-versa. In the next subsection, this analytical solution appears to be a good approximation, even at low rates. It saves computational cost since only boundaries and output points need to be calculated.

\section{Application: Gaussian Data}

The performance of our quantizer is evaluated using the same independent bivariate Gaussian source as in Section II-B. The following quantizers are compared: the optimal UPQ [13], the high-rate optimal UPQ [14], and our embedded UPQ (without the high-rate approximation for the refinement choice). For the optimal UPQ, the simulation has been performed up to a rate of 6 bits only, since for higher rates, the computational cost is too high.

To evaluate the excess distortion due to the high-rate approximation for the refinement choice, the excess distortion over the embedded UPQ is plotted for the embedded UPQ with the high-rate approximation for the refinement choice (see Fig. 3). The excess distortion for the embedding property here is always less than $1 \mathrm{~dB}$ over optimal non-embedded, a price to pay that can, in certain applications, be considered small with regard to its benefits. Finally, the first six embedded UPQ are shown in Fig. 4, using the high-rate approximation for the refinement choice.

\section{CONCLUSION}

This letter describes simple algorithms to design polar quantizers that can be embedded. Two kinds of quantizers have been distinguished: embedded strict polar quantizers where amplitude and phase are quantized separately and embedded unrestricted polar quantizers where amplitude and phase are quantized jointly. For bivariate Gaussian data, this results in a small increase of distortion over when compared to the standard optimal polar quantizers (typically less than $0.2 \mathrm{~dB}$ for SPQ, less than $1 \mathrm{~dB}$ for UPQ). A high-rate approximation for the refinement choice has been introduced and results in negligible excess distortion. This tool is a first step toward efficient bit-plane encoding of polar data, which have applications such as fine-grain scalable parametric audio coding.

\section{REFERENCES}

[1] J. M. Shapiro, "Embedded image coding using zerotrees of wavelet coefficients," IEEE Trans. Signal Process., vol. 41, no. 12, pp. 3445-3462, Dec. 1993.

[2] A. Said and W. A. Pearlman, "A new fast and efficient image codec based on set partitioning in hierarchical trees," IEEE Trans. Circuits Syst. Video Technol., vol. 6, no. 3, pp. 243-250, Jun. 1996.

[3] M. Raad, A. Mertins, and I. Burnett, "Scalable to lossless audio compression based on perceptual set partitioning in hierarchical trees (PSPIHT)," in Proc. IEEE Int. Conf. Acoustics, Speech, Signal Processing, May 2003, vol. 5, pp. 624-627.

[4] R. Vafin and W. B. Kleijn, "Entropy-constrained polar quantization and its application to audio coding," IEEE Trans. Speech Audio Process., vol. 13, no. 2, pp. 220-232, Mar. 2005.

[5] N. G. Kingsbury and T. H. Reeves, "Iterative image coding with overcomplete complex wavelet transforms," in Proc. Conf. Visual Communication and Image Processing, Jul. 2003, pp. 1253-1264.

[6] K.-H. Tzou, "Embedded Max quantization," in Proc. IEEE Int. Conf. Acoustics, Speech, Signal Processing, Apr. 1986, vol. 11, pp. 505-508.

[7] J. Max, "Quantizing for minimum distortion," IRE Trans. Inf. Theory, vol. IT-6, no. 1, pp. 7-12, Mar. 1960.

[8] J. L. Kim, K. Lee, and T. Kim, "Adaptive reconstruction for embedded quantisation," Electron. Lett., vol. 38, no. 18, pp. 1065-1067, Aug. 2002.

[9] H. Brunk and N. Farvardin, "Fixed-rate successively refinable scalar quantizers," in Proc. IEEE Data Compression Conf., 1996, pp. 250-259.

[10] H. Brunk and N. Farvardin, "Embedded trellis coded quantization," in Proc. IEEE Data Compression Conf., 1998, pp. 93-102.

[11] A. Gersho and R. M. Gray, Vector Quantization and Signal Compression. Norwell, MA: Kluwer, 1991.

[12] W. A. Pearlman, "Polar quantization of a complex Gaussian random variable," IEEE Trans. Commun., vol. COMM-27, pp. 892-899, Jun. 1979.

[13] S. G. Wilson, "Magnitude/phase quantization of independent Gaussian variates," IEEE Trans. Commun., vol. COMM-28, pp. 1924-1929, Nov. 1980.

[14] P. F. Swaszek and T. W. Ku, "Asymptotic performance of unrestricted polar quantizers," IEEE Trans. Inf. Theory, vol. 32, no. 2, pp. 330-333, Mar. 1986 